\title{
Efeito dos Ionóforos e do Balanço Eletrolítico da Dieta sobre o Desempenho e a Incidência de Discondroplasia Tibial em Frangos de Corte na Fase Inicial ${ }^{1}$
}

\author{
José Rodrigo Galli Francoㄹ, Alice Eiko Murakami ${ }^{4}$, Márcia Izumi Sakamoto ${ }^{2}$, Elias Nunes \\ Martins $^{4}$, Ivan Moreira 4 , Marli Aparecida dos Santos Pereira ${ }^{3}$
}

RESUMO - O objetivo do trabalho foi avaliar o efeito dos ionóforos e balanços eletrolíticos da dieta (BED) no desempenho e na incidência de discondroplasia tibial (DT) em frangos de corte de 1 a 21 dias de idade. Foram utilizados 1.600 pintos de corte, machos, "Cobb", distribuídos em um delineamento inteiramente casualizado, em esquema fatorial com três níveis de BED (230, 260 e $290 \mathrm{mEq} /$ $\mathrm{kg}$ ) e dois ionóforos (lasalocida e salinomicina), mais dois tratamentos controle (230 e $260 \mathrm{mEq} / \mathrm{kg}$ ), isentos de ionóforos, totalizando oito tratamentos, quatro repetições e 50 aves por unidade experimental. Verificou-se interação entre ionóforos e balanços para o peso aos 21 dias (P21) e consumo de ração (CR). Desdobrando essa interação, observou-se que, com a utilização de lasalocida, houve comportamento quadrático, em função do BED para o P21 e CR, sendo os valores mínimos para essas variáveis obtidos com 261 e $268 \mathrm{mEq} / \mathrm{kg}$, respectivamente. Quando se utilizou salinomicina, não houve efeito do BED. A conversão alimentar (CA) não sofreu influência dos ionóforos e do BED. Na comparação utilizando contrastes, constatou-se melhor P21 das aves com a adição de ionóforos às dietas, todavia, para o CR e CA não foram encontradas diferenças. Na comparação entre ionóforos, as aves que receberam salinomicina apresentaram peso superior àquelas que receberam lasalocida. O uso de salinomicina não mostrou efeito do BED sobre as áreas epifisárias, entretanto, com o uso do lasalocida, o incremento do BED proporcionou aumento linear dessas áreas. Concluiuse que o uso de dietas com lasalocida reduziram o desempenho dos frangos, sendo que as aves que receberam salinomicina apresentaram maior P21 e não sofreram influência do BED na incidência de DT. No entanto, para lasalocida, houve aumento das áreas ósseas, que caracterizam a DT, com o aumento do BED.

Palavras-chave: anticoccidiano, equilíbrio ácido-base, lasalocida, problema de perna, salinomicina

\section{Effect of Ionophores and Acid-Base Balance on Performance and Incidence of Tibial Dyschondroplasia in 21-d Old Broiler Chicks}

\begin{abstract}
The objective of this research was to evaluate the effect of different ionophores and acid-base balance of diets (DEB) on young broiler chicks (one to twenty one days of age) performance and incidence of tibial dyschondroplasia (TD). One thousand and sixty one day old "Cobb" male broilers chickens were allotted to a completely randomized design in $3 \mathrm{x} 2$ factorial arrangement $(\mathrm{DEB}=230,260$ and $290 \mathrm{mEq} / \mathrm{kg}$ x ionophores $=$ lasalocid e salinomicin $)$ and two control treatments $(230$ and $260 \mathrm{mEq} / \mathrm{kg}$ ). The ionophores x DEB interaction, for weight at 21 days (W21) and feed intake (FI), was unrolled and, by using lasalocid, it was observed quadratic effect, in function of DEB levels for W21 and FI. Minimum values for these variables were obtained at levels of 261 and $268 \mathrm{mEq} / \mathrm{kg}$, respectively. When salinomycin was used, DEB effect was observed. Feed: gain ratio was influenced neither by ionophores nor by DEB. In the contrasts comparison, the better W21 was observed when the ionophores were added to the diets, but for the feed intake and feed: gain ratio no differences were observed. In the comparisons between ionophores, the birds fed salinomycin showed better weight than those fed lasalocid. There was no effect of salinomycin on the epiphysis areas, independently of DEB, however, when lasalocid was used, these areas increased, as DEB levels increased. It was concluded that the use of diets with lasalocid reduced the broiler chicks performance, and the birds fed salinomycin showed better W21 and were not affected by DEB on TD incidence. However, when lasalocid was used, the bone areas increase, that characterized TD, as DEB levels increased.
\end{abstract}

Key Words: coccidiostatic, acid-base balance, lasalocid, leg problems, salinomycin

\footnotetext{
${ }^{1}$ Trabalho apresentado ao Departamento de Zootecnia da Universidade Estadual de Maringá como parte das exigências para obtenção do título de Zootecnista.

${ }^{2}$ Aluno do curso de Zootecnia da Universidade Estadual de Maringá. E.mail: zegalli@yahoo.com.br; mizumis@bol.com.br

${ }_{3}^{3}$ Professora Dra. do Departamento de Ciências Morfológicas da UEM. E.mail: madspereira@uem.br

${ }^{4}$ Professor, Dr, Departamento de Zootecnia da UEM. Av. Colombo, 5790 - CEP: 87020-900 - Maringá, PR. E.mail: aemurakami@uem.br; enmartins@uem.br; imoreira@uem.br
} 


\section{Introdução}

Pesquisas têm sido realizadas com o intuito de avaliar a importância dos eletrólitos sobre o desempenho dos frangos de corte. Inúmeros autores têm relatado que o balanço eletrolítico pode influenciar, principalmente o equilíbrio ácido-base $(\mathrm{EAB}) \mathrm{da}$ ave. A manutenção do equilíbrio ácido-base é de grande importância, visto que o frango de corte é uma ave de crescimento extremamente rápido e que drásticas alterações nesse equilíbrio podem acarretar severos danos em seu desempenho.

Edwards Jr. (1984) sugeriu que o balanço eletrolítico para máximo desempenho dos frangos de corte, nas três primeiras semanas, deve ser de aproximadamente $250 \mathrm{mEq} / \mathrm{kg}$.

Ruiz Lopes \& Austic (1993) verificaram que um ótimo balanço está na faixa de 200 a $350 \mathrm{mEq} / \mathrm{kg}$, sendo influenciado pelo $\mathrm{P}$ e $\mathrm{K}$, além do $\mathrm{Na}$ e $\mathrm{Cl}$. Contudo, esses autores não observaram influência definitiva do equilíbrio ácido-base sobre o ganho de peso das aves estudadas.

Oviedo-Rondon et al. (1999), avaliando frangos de corte na fase inicial ( 0 - 21 dias), observaram que o melhor desempenho produtivo das aves foi obtido utilizando-se níveis de balanços eletrolíticos de 246 e $315 \mathrm{mEq} / \mathrm{kg}$ e exigência de $\mathrm{Na}$ de $\mathrm{Cl}$ de 0,28 e $0,25 \%$, respectivamente.

Murakami et al. (2000), utilizando dietas de baixa proteína e balanços eletrolíticos de 200 a $320 \mathrm{mEq} / \mathrm{kg}$ para frangos de corte de 1 a 21 dias de idade, constataram efeito linear crescente do balanço sobre o peso aos 21 dias e consumo de ração.

O desequilíbrio de cátions e ânions na dieta, além de afetar o desempenho das aves, pode ter influência na incidência de problemas de pernas em pintos. Leach \& Neshein $(1965,1972)$, estudando pintos alimentados com dietas purificadas, descreveram anomalias da cartilagem epifisária da tíbia, denominadas de discondroplasia tibial, concluindo que o aparecimento da discondroplasia apresentava uma relação positiva com o conteúdo de cloro, o que também foi confirmado por Riddel (1975), utilizando dietas práticas, e Sauveur \& Mongin (1978), usando os dois tipos de dietas.

A discondroplasia tibial é uma lesão comum na zona de crescimento ósseo em frangos, patos e perus. Caracteriza-se pela persistência de uma massa cartilaginosa anormal na região da metáfise, imediatamente abaixo da zona ou placa de crescimento, principalmente na tíbia. Nessa lesão, não ocorrem hipertrofia, mineralização, invasão vascular e remoção da cartilagem da zona hipertrófica, entretanto, a sua patogênese não está bem esclarecida, porém sua incidência pode ser influenciada pela genética, pelo manejo e pela nutrição (Whitehead, 1992).

Halley et al. (1987) observaram alta correlação entre a discondroplasia e o desequilíbrio ácido-base, indicando a existência do efeito da manipulação do conteúdo mineral da dieta sobre a capacidade tampão do sangue, o que pode afetar funções como a mineralização óssea.

O mecanismo exato de influência do equilíbrio ácido-base sobre a calcificação do osso não é bem definido, entretanto, Mongin \& Sauveur (1977) sugerem o envolvimento com o metabolismo da vitamina D. A acidose metabólica pode prejudicar a maturação da cartilagem por meio da alteração da síntese renal de 1-25 dihidroxicolecalciferol [1-25( $\left.\left(\mathrm{OH}_{2}\right)\right]$ e do 25-hidroxi-colecalciferol no fígado.

Bushinsky (1995), estudando a acidose metabólica, tanto in vivo quanto in vitro, afirma que a mesma tem efeitos significativos sobre os minerais ósseos. Em culturas ósseas e em células ósseas, a acidose metabólica não somente causou significante aumento na saída de cálcio, mas também estimulou a reabsorção óssea pelos osteoclastos, inibiu a formação óssea pelos osteoblastos e diminuiu a formação de nódulos mineralizados nos ossos (Bushinsky \& Sessler, 1992; Krieger, 1992; Sprague, 1994).

$\mathrm{Na}$ busca do balanço eletrolítico da dieta para minimizar a incidência da discondroplasia tibial, Hulan et al. (1987) observaram que quando o balanço $\mathrm{Na}+\mathrm{K}-\mathrm{Cl}$ está entre 155 e $300 \mathrm{mEq} / \mathrm{kg}$, não havendo influência negativa sobre o ganho de peso, conversão alimentar e discondroplasia tibial.

Murakami et al. (2000), ao utilizarem balanço eletrolítico de 200 a $320 \mathrm{mEq} / \mathrm{kg}$, para frangos de corte de 1 a 21 dias de idade, observaram diminuição na zona de cartilagem hipertrófica da tíbia, que caracteriza a discondroplasia, com o aumento do balanço, obtendo a menor área, quando o balanço eletrolítico utilizado era de $253 \mathrm{mEq} / \mathrm{kg}$. Da mesma forma, houve redução da área total da epífise tibial com o aumento do balanço, sendo que a menor área observada ocorreu em $248 \mathrm{mEq} / \mathrm{kg}$.

A determinação do balanço eletrolítico da dieta ótimo é dificultada pelo fato de a exigência de eletrólitos ser influenciada pela utilização de ionóforos nas rações. Estes produtos utilizados na prevenção e 
no combate à coccidiose aviária têm efeito sobre o balanço de minerais, podendo alterar a disponibilidade dos mesmos e, conseqüentemente, a exigência nutricional para o adequado crescimento da ave (Jensen, 1980). Segundo Rutz et al. (1999), a maior parte dos ionóforos aumenta a permeabilidade das membranas aos íons $\mathrm{H}^{+}$, um fator significativo no equilíbrio ácido-base.

De acordo com Austic \& Smith (1980), ionóforo é um termo genérico aplicado a um número de compostos do grupo dos antibióticos denominados poliésteres, contendo um radical carboxílico, o qual facilita a difusão de íons através de barreiras lipídicas, como as membranas celulares.

Por apresentarem diferentes estruturas moleculares, os ionóforos não apresentam a mesma afinidade entre todos os íons. O lasalocida, de acordo com a Roche (1994), é um ionóforo bivalente, produzido pela fermentação do Streptomyces lasaliensis. Ao contrário dos outros ionóforos, é o único que atua nos íons bivalentes $\left(\mathrm{Ca}^{++} \mathrm{e} \mathrm{Mg}^{++}\right)$, além de atuar nos íons monovalentes $\left(\mathrm{Na}^{+}\right.$e K $\left.{ }^{+}\right)$. Como substância ionofórica, apresenta predileção pelos cátions $\mathrm{Na}, \mathrm{K}$ e, especialmente, $\mathrm{Ca}$ (Pressman, 1976). A salinomicina, no entanto, é obtida através da fermentação de uma cepa de Streptomyces albus, apresentando a propriedade de formar complexos com íons alcalinos (Pfizer, 1994). Como substância ionofórica, apresenta alta afinidade pelos íons $\mathrm{Na}^{+} \mathrm{e} \mathrm{K}$ (Ferreira \& Dell'Porto, 1999).

Esses ionóforos, além monensina, são conhecidos por influenciarem as respostas nutricionais nos frangos (Pesti et al., 1999). Alterações no desempenho das aves podem ainda estar relacionadas ao fato de os ionóforos poderem afetar os processos da membrana celular de células eucarióticas e de diversas organelas intracelulares (como a mitocôndria), especialmente os sistemas dependentes de gradiente elétrico, excitabilidade e regulação osmótica. Além disso, as células do intestino delgado seriam o provável alvo inicial dos ionóforos, que poderiam alterar a absorção de aminoácidos e açúcares (Bergen \& Bates, 1984; Novilla, 1992).

A monensina ou o lasalocida altera a disponibilidade de minerais absorvidos pela mucosa intestinal, bem como a deposição tecidual de minerais bivalentes, pois o transporte desses minerais passa a ser comprometido, direta ou indiretamente, pelo mecanismo de ação desses ionóforos, entretanto, a direção dessas alterações não pode ser predita (Elsasser, 1984).

Wheelhouse \& Groves (1985) afirmam, de modo geral, que a utilização de ionóforos, adicionados às rações, promove melhor ganho de peso para os frangos de corte, mesmo em condições de infestações experimentais. No entanto, Rosado (1988) constatou que a utilização de coccidiostáticos ionóforos nas rações para frangos de corte até 28 dias de idade não proporciona ganho de peso significativamente diferente das aves alimentadas com ração isenta dos mesmos, sem infestação experimental.

Karunajeewa \& Barr (1988) concluíram que a inclusão de $90 \mathrm{mg} / \mathrm{kg}$ de monensina ou lasalocida nas dietas de frangos de corte de 1 a 42 dias de idade, empregando-se um balanço eletrolítico de 125 a $205 \mathrm{mEq} / \mathrm{kg}$, não altera a exigência de eletrólitos na obtenção do balanço eletrolítico para ótimo desempenho.

Hooge (1995) observou que os ionóforos podem ter a sua ação aumentada ou potencializada pelos eletrólitos da dieta. Em diversos estudos, Hooge et al. (1999) observaram efeito sinérgico benéfico, quando um ionóforo (monensina ou salinomicina) foi fornecido em combinações com níveis de $0,2 \%$, ou superiores, de bicarbonato de sódio. Os autores denominaram esse efeito como potencializador de ionóforo, uma vez que, além de reduzir o escore de lesões causadas por coccidiose e mortalidade, também melhorou o desempenho e a qualidade da carcaça dos frangos de corte.

Todavia, comprovada existência da interação entre ionóforos e os eletrólitos, acredita-se que esses agentes anticoccidianos possam ter efeito, direto ou indireto, na incidência de discondroplasia tibial, entretanto, dentro da literatura consultada, não foram encontrados dados que comprovem essa suposição.

Devido à importância da utilização de anticoccidianos ionóforos no combate à coccidiose aviária e suas inter-relações com os eletrólitos, com influência na obtenção do balanço eletrolítico ideal para ótimo desempenho das aves, com menor incidência de discondroplasia tibial, e por existirem inúmeras divergências na literatura em relação à ação desses fatores, realizou-se este trabalho.

Assim, objetivou-se avaliar o efeito dos ionóforos e balanços eletrolíticos da dieta no desempenho e na incidência da discondroplasia tibial em frangos de corte de 1 a 21 dias de idade.

\section{Material e Métodos}

O experimento foi realizado no Aviário da Fazenda Experimental de Iguatemi e no Laboratório de Histologia, da Universidade Estadual de Maringá. 
Foram utilizados 1.600 pintos de corte, machos da linhagem "Cobb", de um dia de idade. As aves foram alojadas em um galpão convencional de 30 metros de comprimento, por oito metros de largura, com cobertura de telha francesa e lanternim, piso de concreto e paredes laterais de alvenaria com $0,40 \mathrm{~m}$ de altura, completadas com tela de arame até o telhado. O galpão é dividido em boxes de $5,1 \mathrm{~m}^{2}$, onde foram alojadas 50 aves e a cama utilizada foi do tipo maravalha.

Durante os primeiros dias, foram utilizados comedouros do tipo bandejas de $0,50 \mathrm{~m}$ x $0,40 \mathrm{~m} \times$ $0,05 \mathrm{~m}$, bebedouros tipo copo de pressão até o $3 \underline{0}$ dia e, depois, substituídos gradativamente pelo comedouro tubular. Em cada "box" foi colocada uma lâmpada de 350 watts, para aquecimento inicial das aves. $\mathrm{O}$ programa de iluminação utilizado foi o contínuo, durante a primeira semana, e de 20 horas de luz e quatro de escuridão diárias, até o final do experimento.

As dietas foram formuladas atendendo às exigências da linhagem e a composição dos ingredientes, de acordo com Rostagno et al. (2000), todas à base de milho e farelo de soja, isocalóricas, isoprotéicas, isoaminoacídicas para metionina + cistina e lisina, isocálcicas e isofosfóricas (Tabela 1).

Os anticoccidianos ionóforos foram adicionados segundo a recomendação do produto. $\mathrm{O}$ anticoccidiano ionóforo lasalocida foi adicionado na proporção de $600 \mathrm{~g} / \mathrm{t}$, constituído de $15 \%$ do lasalocida sódica + veículo, fornecendo $90 \mathrm{mg}$ de lasalocida por $\mathrm{kg}$ de ração. Para a salinomicina, adicionou-se o produto anticoccidiano na proporção de $550 \mathrm{~g} / \mathrm{t}$, sendo o produto constituído de $12 \%$ de salinomicina + veículo, fornecendo $66 \mathrm{mg}$ de salinomicina por $\mathrm{kg}$ de ração. Os suplementos vitamínico e mineral foram formulados especialmente para o trabalho, não contendo qualquer anticoccidiano.

O balanço eletrolítico das dietas experimentais (Tabela 1) foi calculado segundo Mongin (1980), pela fórmula: $\mathrm{N}^{\circ}$ de Mongin $(\mathrm{NM})=\mathrm{mEqNa}^{+}+\mathrm{mEqK}^{+}$$\mathrm{mEqCl}^{-}(\mathrm{mEq} / \mathrm{kg})$.

Para o cálculo do NM, a partir de valores percentuais dos eletrólitos, foi empregada a seguinte fórmula: $\mathrm{N}^{\mathrm{o}} \mathrm{de}$ Mongin $=\left[\left(\% \mathrm{Na}^{+} \times 10000 / 22,990^{*}\right)+\left(\% \mathrm{~K}^{+} \times 10000 /\right.\right.$ $\left.39,102 *)-\left(\% \mathrm{Cl}^{-} \times 10000 / 35,453 *\right)\right] \quad(*$ Equivalente grama do $\mathrm{Na}, \mathrm{K}$ ou $\mathrm{Cl}$, respectivamente).

A obtenção dos níveis experimentais de balanço eletrolítico foi feita por meio da adição de $\mathrm{NaHCO}_{3}$ às dietas experimentais.

Os dados monitorados foram peso aos 21 dias (P21), consumo de ração (CR), conversão alimentar
(CA) e a mortalidade, cujas causas foram determinadas por necropsia.

O delineamento experimental utilizado foi inteiramente casualizado, em esquema fatorial em três níveis de balanço eletrolítico $(230,260$ e $290 \mathrm{mEq} / \mathrm{kg}$ ) e dois ionóforos (lasalocida e salinomicina), mais dois tratamentos controles ( 230 e $260 \mathrm{mEq} / \mathrm{kg}$ ), isentos e ionóforos, totalizando oito tratamentos com quatro repetições e 50 aves por unidade experimental.

Os dados obtidos foram submetidos à análise de regressão polinomial, em função dos níveis de balanço eletrolítico e as comparações entre os ionóforos e os tratamentos controles foram feitas por meio de contrastes.

Para avaliar a incidência à discondroplasia tibial, duas aves de cada unidade experimental foram sacrificadas aos 21 dias de idade, retirando-se a tíbia da perna esquerda, que foi fixada em solução de Bouin.

A descalcificação do material foi realizada com ácido nítrico e fluroglucina (Solução de Haug) para evitara hidrólise e o entumecimento do tecido ósseo. Após a descalcificação, o osso foi submetido à rotina histológica, para inclusão em parafina (Beçak \& Paulete, 1976). Os cortes foram feitos com micrótomo rotativo a $5 \mathrm{~mm}$ de espessura e corados com Hematoxilina-Eosina, para observação da zona do disco epifisário e mensurações das áreas para caracterização da incidência à discondroplasia tibial.

Para análise das lâminas da cartilagem epifisária tibial, foram consideradas três regiões distintas caracterizadas pela aparência morfológica: zona de repouso, zona de cartilagem em proliferação e zona de cartilagem hipertrófica. A zona de cartilagem calcificada foi considerada como o limite inferior para determinação do espessamento da zona hipertrófica, na caracterização da lesão, segundo Ridell (1975) e Thorp et al. (1993).

Foi necessário idealizar uma metodologia de determinação de áreas da cartilagem do corte histológico do perfil longitudinal da tíbia, por meio de um software para análises de imagens (ImagePro $\left.{ }^{\circledR}\right)$. Para este processo, as lâminas histológicas confeccionadas foram "scaneadas" utilizando o scanner HP ScanJet II v.2.5, utilizando-se o software o Paint Shop Pro V.5.0 para tratamento de imagem. A imagem foi digitalizada utilizando o modo "branco e preto" com qualidade de foto, com as seguintes características: brilho (variável entre 125 e 145), contraste 185 e a escala utilizada de $800 \%$.

As imagens gravadas foram submetidas ao 
Tabela 1 - Composição percentual e calculada das dietas experimentais

Table 1 - Percentual and calculated composition of the experimental diets

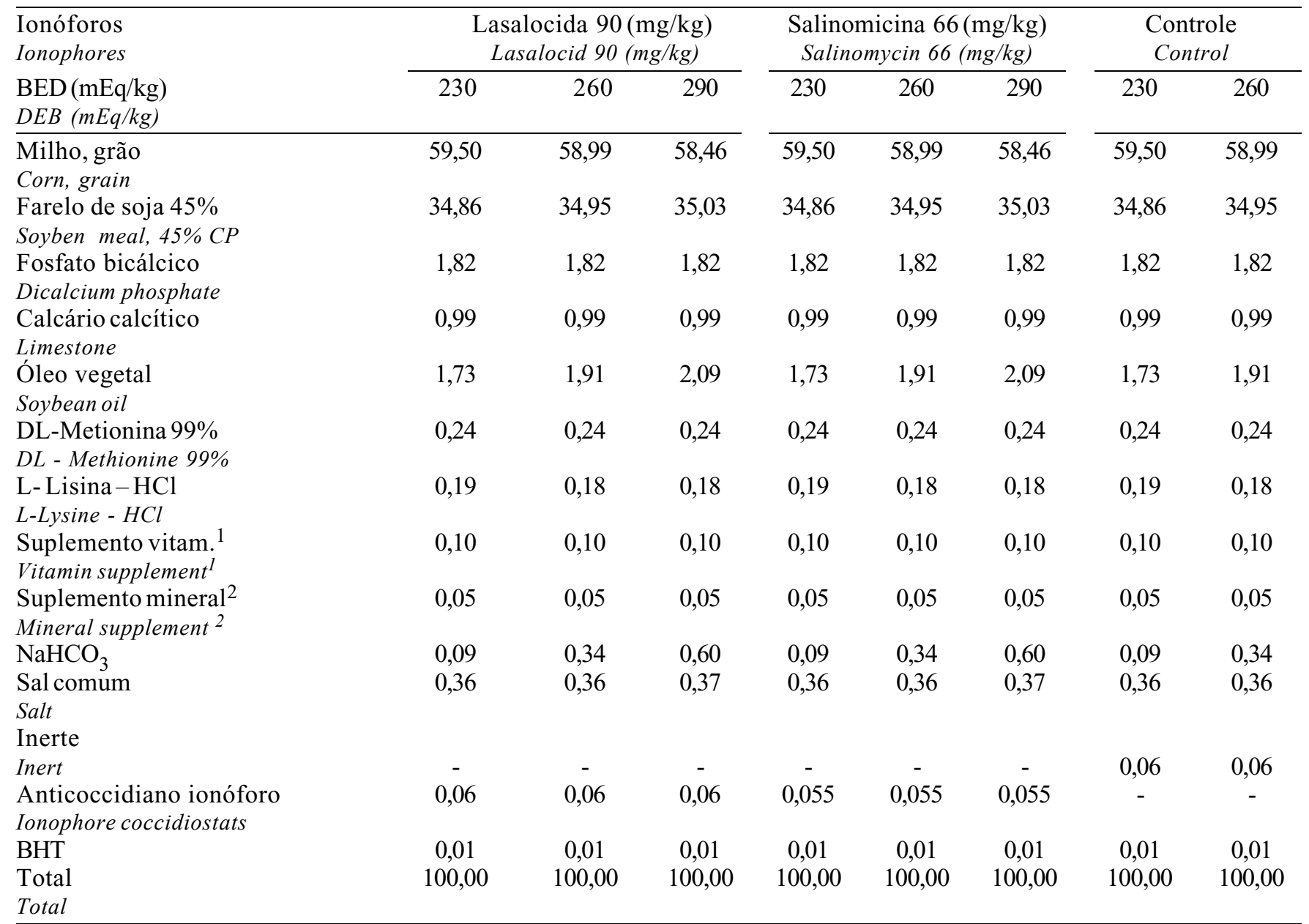

Valores calculados

Calculated values

\begin{tabular}{|c|c|c|c|c|c|c|c|c|}
\hline $\begin{array}{l}\text { Energia metabolizável }(\mathrm{kcal} / \mathrm{kg}) \\
\text { Metabolizable energy }(\mathrm{kcal} / \mathrm{kg})\end{array}$ & 3.000 & 3.000 & 3.000 & 3.000 & 3.000 & 3.000 & 3.000 & 3.000 \\
\hline Proteína bruta $(\%)$ & 21,292 & 21,292 & 21,292 & 21,292 & 21,292 & 21,292 & 21,292 & 21,292 \\
\hline $\begin{array}{l}\text { Crude protein } \\
\text { Met }+\operatorname{cis}(\%)\end{array}$ & 0,897 & 0,897 & 0,897 & 0,897 & 0,897 & 0,897 & 0,897 & 0,897 \\
\hline $\begin{array}{l}\text { Met }+ \text { Cys } \\
\text { Lisina }(\%) \\
\text { Lysine }\end{array}$ & 1,263 & 1,263 & 1,263 & 1,263 & 1,263 & 1,263 & 1,263 & 1,263 \\
\hline $\begin{array}{l}\text { Cálcio (\%) } \\
\text { Calcium }\end{array}$ & 0,960 & 0,960 & 0,960 & 0,960 & 0,960 & 0,960 & 0,960 & 0,960 \\
\hline $\begin{array}{l}\text { P disponível (\%) } \\
\text { Available } P\end{array}$ & 0,450 & 0,450 & 0,450 & 0,450 & 0,450 & 0,450 & 0,450 & 0,450 \\
\hline $\begin{array}{l}\text { Sódio }(\%) \\
\text { Sodium }\end{array}$ & 0,209 & 0,278 & 0,347 & 0,209 & 0,278 & 0,347 & 0,209 & 0,278 \\
\hline $\begin{array}{l}\text { Cloro }(\%) \\
\text { Chloride }\end{array}$ & 0,250 & 0,250 & 0,250 & 0,250 & 0,250 & 0,250 & 0,250 & 0,250 \\
\hline Potássio (\%) & 0,820 & 0,820 & 0,820 & 0,820 & 0,820 & 0,820 & 0,820 & 0,820 \\
\hline
\end{tabular}

Potassium

${ }^{1}$ Suplemento vitamínico para aves. Níveis de garantia por kg de produto: Vit. A - 7.000.000 Ul; Vit. $D_{3}-2.200 .000$ Ul; Vit. E - 11.000 mg; Vit. $K_{3}-1.600 \mathrm{mg}$; Vit. $B_{1}-2.000 \mathrm{mg}$; Vit. $B_{2}-5.000 \mathrm{mg}$; Vit. $B_{6}-3.000 \mathrm{mg}$; Vit. $B_{12}-12.000 \mathrm{mg}$; Ác. fólico - $800 \mathrm{mg}$; Ác. pantotênico - $13.000 \mathrm{mg}$; Antioxidante - $100.000 \mathrm{mg}$; Véículo q.s.p. - $1.000 \mathrm{~g}$.

${ }^{2}$ Suplemento mineral. Níveis de garantia por kg de produto: Fe - $100.000 \mathrm{mg} ; \mathrm{Mn}-140.000 \mathrm{mg} ; \mathrm{Zn}-100.000 \mathrm{mg} ; \mathrm{Cu}-16.000 \mathrm{mg}$; I - $2.400 \mathrm{mg}$; Se - $400 \mathrm{mg}$; Veículo q.s.p. - $1.000 \mathrm{~g}$.

1 Vitamin supplement. Composition per kg of product: Vit. A - $7.000 .000 \mathrm{IU}$; Vit. $D_{3}-2.200 .000 \mathrm{UI}$; Vit. E - $11.000 \mathrm{mg}$; Vit. $K_{3}-1.600 \mathrm{mg} ; \mathrm{Vit}$. B - $_{1} .000 \mathrm{mg}$; Vit. $B_{2}-5.000 \mathrm{mg}$; Vit. $B_{6}-3.000 \mathrm{mg}$; Vit. $B_{12}-12.000 \mathrm{mg}$; Folic acid - $800 \mathrm{mg}$; Pantothenic acid - $13.000 \mathrm{mg}$; Antioxidant - $100.000 \mathrm{mg}$; Vehicle q.s.p. $1.000 \mathrm{~g}$.

${ }^{2}$ Mineral supplement. Composition per kg of product: Fe - 100.000 mg; Mn - 140.000 mg; Zn - $100.000 \mathrm{mg}$; Cu - $16.000 \mathrm{mg} ; \mathrm{I}-2.400 \mathrm{mg}$; Se - $400 \mathrm{mg}$; Vehicle q.s.p. $-1.000 \mathrm{~g}$.

R. Bras. Zootec., v.33, n.1, p.135-145, 2004 
analisador de imagens, onde foi realizada prévia calibração em milímetros do equipamento e determinadas e medidas de três áreas: 1 . Zona de cartilagem em proliferação ou placa de crescimento, 2. Zona de cartilagem hipertrófica e 3. Área total da epífise, como ilustra a Figura 1.

Uma vez que os valores das áreas avaliadas não tinham distribuição normal, estes dados foram analisados por meio da metodologia de Modelos Lineares Generalizados (GLM), admitindo-se distribuição gama e função de ligação recíproca.

\section{Resultados e Discussão}

$\mathrm{Na}$ comparação entre ionóforos, as aves que receberam salinomicina apresentaram peso superior $(\mathrm{P} \leq 0,05)$ àquelas que receberam lasalocida (Tabela 2), evidenciando melhor eficácia da salinomicina e confirmando que o lasalocida, devido provavelmente à sua composição molecular e, conseqüentemente, seu mecanismo de ação, pode comprometer o transporte e a disponibilidade dos minerais que são absorvidos pela mucosa intestinal, além de interferir na distribuição normal e bioatividade dos íons nos tecidos e células, como sugere Elsasser (1984).

Com o aumento do balanço eletrolítico da dieta, através da elevação nos níveis de sódio, o lasalocida pode ter sua ação potencializada (Hooge et al., 1995), pois apresenta alta afinidade por este eletrólito. Ao aumentar o transporte de eletrólitos, ligados a sua molécula, o lasalocida teria indisponibilizado-os aos diversos processos relacionados com a absorção de aminoácidos e açúcares no intestino delgado, uma vez que as células do intestino seriam o provável alvo inicial dos ionóforos (Bergen \& Bates, 1984; Novilla, 1992).

A absorção de glicose e aminoácidos é dependente do processo de transporte ativo desempenhado pela bomba Na-K-ATPase. Aminoácidos ácidos apresentam-se mais dependentes da concentração de sódio para serem absorvidos e monossacarídeos como a glicose e galactose dependem da presença de "carreadores" ativados pelo sódio(Vieira \& Rutz, 2002).

O lasalocida, ao se ligar ao sódio, bem como a outros eletrólitos, transportá-los e provavelmente indisponibilizá-los, pode ter alterado alguns processos dependentes dos mesmos, como os relacionados à absorção de nutrientes, outros minerais, manutenção de gradiente elétrico e regulação osmótica. A
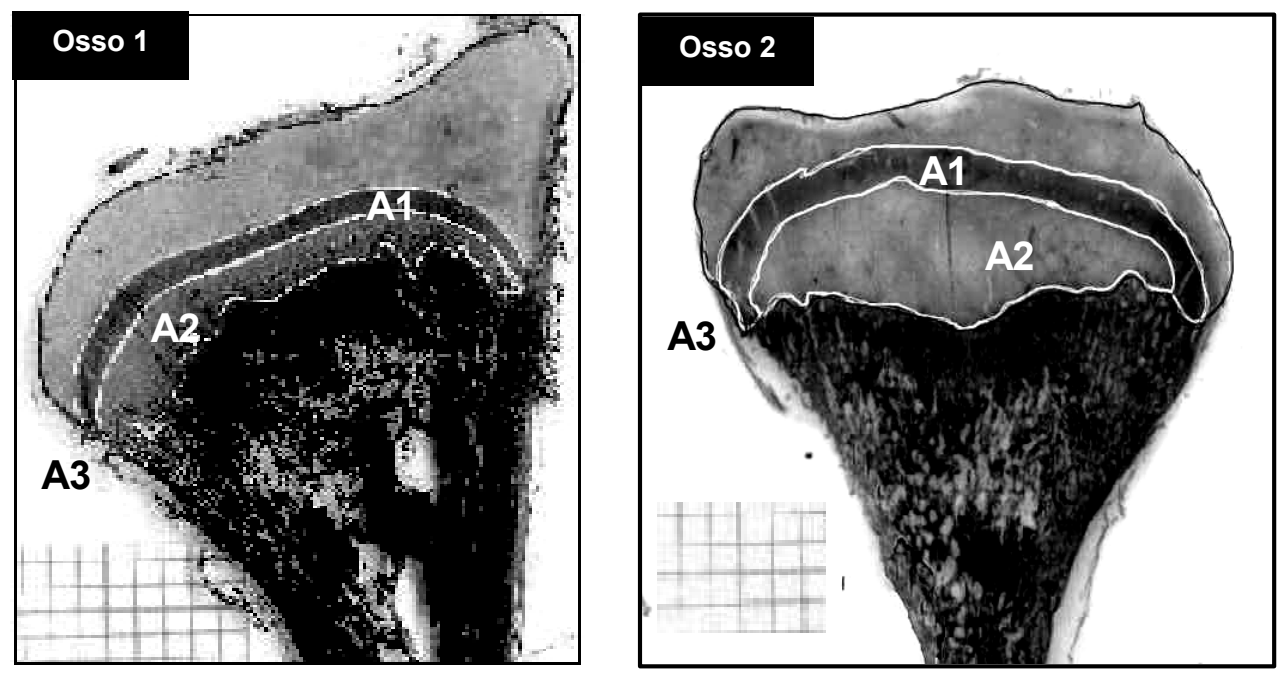

Figura 1 - Corte histológico da epífise da tíbia de um frango de corte aos 21 dias de idade sem discondroplasia (osso um) e com a lesão (osso dois), demonstrando as áreas medidas na caracterização da lesão, sendo elas: zona de cartilagem em proliferação ou placa de crescimento (A1), zona de cartilagem hipertrófica (A2) e área total da epífise (A3).

Figure 1 - Histological cut of the tibia epiphysis of 21-day old broilers chickens, without dyschondroplasia (bone one) and with the lesion (bone two), demonstrating the measured areas in the characterization of the lesion: growth plate (A1), hypertrophic cartilage zone (A2) and total area of epiphysis (A3). 
irregular atividade desses processos fisiológicos, possivelmente, teria causado o menor peso às aves que receberam o lasalocida.

Comparando-se os ionóforos aos tratamentos controles, verificou-se $(\mathrm{P} \leq 0,05)$ melhor peso aos 21 dias das aves, quando houve a adição de lasalocida ou salinomicina nas dietas, em relação às aves que receberam as dietas controles, sem adição de ionóforos (Tabela 3). No entanto, para o consumo de ração e conversão alimentar, não foram encontradas diferenças $(\mathrm{P}>0,05)$.

A melhora no peso aos 21 dias das aves está de acordo com os resultados obtidos por Hooge et al. (1995), os quais verificaram que a adição de ionóforos (monensina ou salinomicina), com níveis de $0,2 \%$, ou superiores de bicarbonato de sódio, melhora o desempenho dos frangos de corte, da mesma forma que Wheelhouse \& Groves (1985) afirmam que a adição dos ionóforos promovem melhor ganho de peso, mesmo em condições de infestação experimental.
O efeito positivo dos ionóforos sobre o peso das aves discorda do resultado obtido por Rosado (1988), que não observou efeito da adição de ionóforos sobre o ganho de peso dos frangos de corte.

Os efeitos dos ionóforos sobre o desempenho das aves variam de acordo com o ionóforo utilizado, a idade da ave, a concentração utilizada nas rações e o fato de a ave apresentar ou não infestação por eimeria .

Verificou-se a presença de interação $(\mathrm{P} \leq 0,05)$ entre ionóforos e níveis de balanços eletrolíticos, para o peso aos 21 dias e consumo de ração. Desdobrando-se a interação, observou-se que, com o uso de lasalocida, houve efeito quadrático dos níveis de balanços eletrolíticos sobre o peso aos 21 dias ( $\hat{\mathrm{Y}}=2,7649-0,0135122 \mathrm{BED}+0,0000258485 \mathrm{BED}^{2}$; $\mathrm{r}^{2}$ ajust. $\left.=0,36\right)($ Figura 2) e sobre o consumo de ração ( $\hat{Y}=2,92944-0,0120219 B E D+0,0000224098 B^{2} D^{2}$; $\mathrm{r}^{2}$ ajust. $\left.=0,46\right)($ Figura 3$)$. Os valores mínimos para o peso aos 21 dias e consumo de ração são preditos

Tabela 2 - Desempenho dos frangos de corte aos 21 dias de idade, submetidos a dietas com diferentes ionóforos

Table 2 - Performance of broiler chickens in the starting period, fed diets with different ionophores

\begin{tabular}{|c|c|c|c|}
\hline \multirow{3}{*}{$\begin{array}{l}\text { Variáveis } \\
\text { Variables }\end{array}$} & \multicolumn{2}{|c|}{$\begin{array}{l}\text { Ionóforos } \\
\text { Ionophores }\end{array}$} & \multirow[t]{3}{*}{$\mathrm{CV}^{1}(\%)$} \\
\hline & Lasalocida & Salinomicina & \\
\hline & Lasalocid & Salinomycin & \\
\hline$\overline{\text { Peso aos } 21 \text { dias (g) }}$ & $1.014,60 *$ & $1.029,94$ & 1,34 \\
\hline 21-day body weight & & & \\
\hline $\begin{array}{l}\text { Consumo de ração }(\mathrm{g}) \\
\text { Feed intake }\end{array}$ & $1.331,98^{* *}$ & $1.346,59$ & 1,69 \\
\hline $\begin{array}{l}\text { Conversão alimentar }(\mathrm{g} / \mathrm{g}) \\
\text { Feed:gain ratio }\end{array}$ & 1,374 & 1,366 & 1,19 \\
\hline
\end{tabular}

1 Coeficiente de variação (Coefficient of variation).

* $\hat{\mathrm{Y}}=2,7649-0,0135122 \mathrm{BED}+0,0000258485 B E D^{2} ; \mathrm{R}^{2}$ ajust. $=0,36$.

$* * \hat{\mathrm{Y}}=2,92944-0,0120219 B E D+0,0000224098 B E D^{2} ; R^{2}$ ajust. $=0,46$.

Tabela 3 - Peso (P.21 dias), consumo de ração (CR) e conversão alimentar (CA) dos frangos de corte aos 21 dias de idade, submetidos a dietas com ionóforos e balanços eletrolíticos da dieta (BED) e sem ionóforos

Table 3 - Body weight, feed intake and feed:gain ratio of broilers fed diets with ionophores and electrolyte balances and without ionophores, in the starting period

\begin{tabular}{lccc}
\hline $\begin{array}{l}\text { Tratamentos } \\
\text { Treatments }\end{array}$ & $\begin{array}{c}\text { P. } 21 \text { dias }(\mathrm{g})^{1} \\
\text { 21-day body weight }\end{array}$ & $\begin{array}{c}\text { CR }(\mathrm{g}) \\
\text { Feed intake }\end{array}$ & $\begin{array}{c}\text { CA }(\mathrm{g} / \mathrm{g}) \\
\text { Feed:gain ratio }\end{array}$ \\
\hline $\begin{array}{l}\text { Ionóforos } \\
\text { Ionophores } \\
\begin{array}{l}\text { Controle } \\
\text { Control }\end{array}\end{array}$ & 1022,26 & 1339,27 & 1,370 \\
\hline
\end{tabular}

${ }_{1}^{1}$ Contrastes $(P \leq 0,05)$.

${ }^{1}$ Contrasts $(P \leq .05)$. 
para os níveis de balanços eletrolíticos de 261 e 268 $\mathrm{mEq} / \mathrm{kg}$, respectivamente.

Estes resultados divergem de Hullan et al. (1987) e Ruiz-Lopes et al. (1993), os quais constataram que ótimo balanço eletrolítico está na faixa de 155 a 300 e 200 a $350 \mathrm{mEq} / \mathrm{kg}$, respectivamente. Além disso, Murakami et al. (2000) observaram comportamento linear crescente do balanço sobre o peso aos 21 dias e consumo de ração, o que não foi observado no presente trabalho. Entretanto, esses autores não avaliaram o efeito dos níveis do balanço eletrolítico em dietas contendo ionóforos.

Os resultados obtidos demonstram que a adição do ionóforo lasalocida à alimentação das aves pode alterar a exigência de eletrólitos na obtenção do balanço eletrolítico para ótimo desempenho das mesmas, contrariando Karunajeewa \& Barr (1988), os quais concluíram que a utilização de lasalocida, com o incremento dos níveis do balanço eletrolítico de 125 a $205 \mathrm{mEq} / \mathrm{kg}$, não influencia essa exigência. Contudo, os níveis do balanço eletrolítico estudados por esses autores apresentam-se inferiores aos avaliados neste trabalho.

Quando se utilizou a salinomicina, não houve efeito $(\mathrm{P}>0,05)$ do balanço eletrolítico, demonstrando que a salinomicina, através de seu mecanismo de ação, não altera a exigência de eletrólitos, provavelmente, por não interferir de forma significativa em seu transporte, disponibilidade e bioatividade.

A conversão alimentar não sofreu influência $(\mathrm{P}>0,05)$ dos ionóforos e dos níveis de balanços

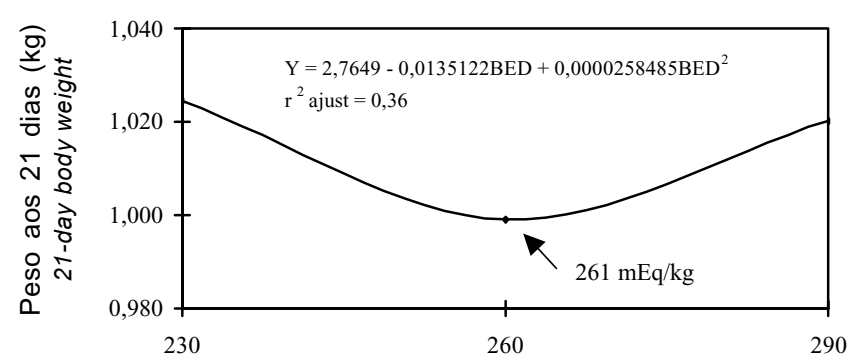

Balanço eletrolítico da dieta $(\mathrm{mEq} / \mathrm{kg})$ Electrolyte balance of diet

Figura 2 - Efeito do balanço eletrolítico, em dietas contendo lasalocida, sobre o peso dos frangos de corte aos 21 dias de idade.

Figure 2 - Effect of electrolyte balance in diets with lasalocid on body weight in broilers chickens at 21 days of age. eletrolíticos, contrariando o que foi constatado por Edwards Jr. (1984) e Oviedo-Rondon et al. (1999), os quais afirmam que melhor desempenho das aves, nessa fase, pode ser influenciado pelo balanço eletrolítico, sugerindo a utilização de 250 e 246 ou $315 \mathrm{mEq} / \mathrm{kg}$, respectivamente.

$\mathrm{Na}$ avaliação da incidência de discondroplasia tibial, constatou-se que, com a utilização da salinomicina, não houve efeito $(\mathrm{P}>0,05)$ dos níveis de balanços eletrolíticos sobre as áreas epifisárias ósseas mensuradas, que caracterizam a lesão.

Da mesma forma que para o peso aos 21 dias e o consumo de ração, a análise das áreas epifisárias da tíbia indicou interação $(\mathrm{P} \leq 0,05)$ entre o lasalocida e os níveis do balanço eletrolítico.

Com o uso de lasalocida, o incremento nos níveis de balanço eletrolítico provocou aumentos lineares $(\mathrm{P} \leq 0,05)$ das áreas da placa de crescimento ( $\mathrm{Y}=1 /$ 0,1644 - 0,0004114BED), zona de cartilagem hipertrófica $(\mathrm{Y}=1 / 0,1532$ - 0,0004653BED) e área epifisária total ( $\mathrm{Y}=1 /$ 0,03205-0,00008302BED), como ilustra a Figura 4.

Esses resultados evidenciam que a utilização do lasalocida pode dificultar a tentativa de reduzir as áreas ósseas relacionadas à ocorrência da discondroplasia, por meio da manipulação do balanço eletrolítico, uma vez que, segundo Murakami et al. (2000), a zona de cartilagem hipertrófica e área total da epífise tibial podem ser reduzidas com a utilização de um balanço eletrolítico da dieta de 253 e $248 \mathrm{mEq} / \mathrm{kg}$,

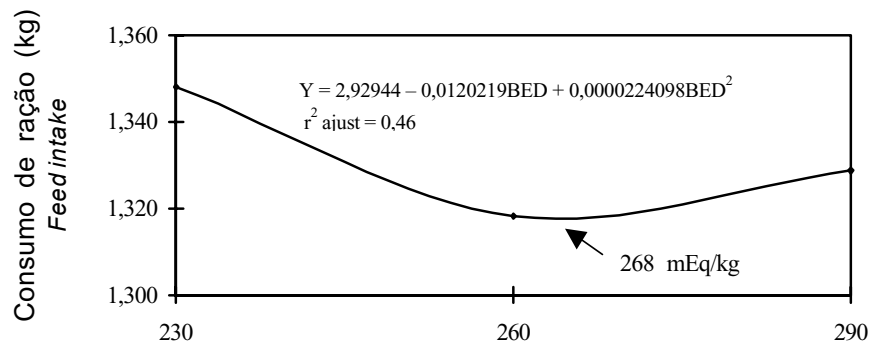

Balanço eletrolítico da dieta $(\mathrm{mEq} / \mathrm{kg})$ Electrolyte balance of diet

Figura 3 - Efeito do BED, em dietas contendo lasalocida, sobre o consumo de ração dos frangos de corte aos 21 dias de idade.

Figure 3 - Effect of the electrolyte balance of diets with lasalocid on feed intake in broilers chickens in the starting period. 

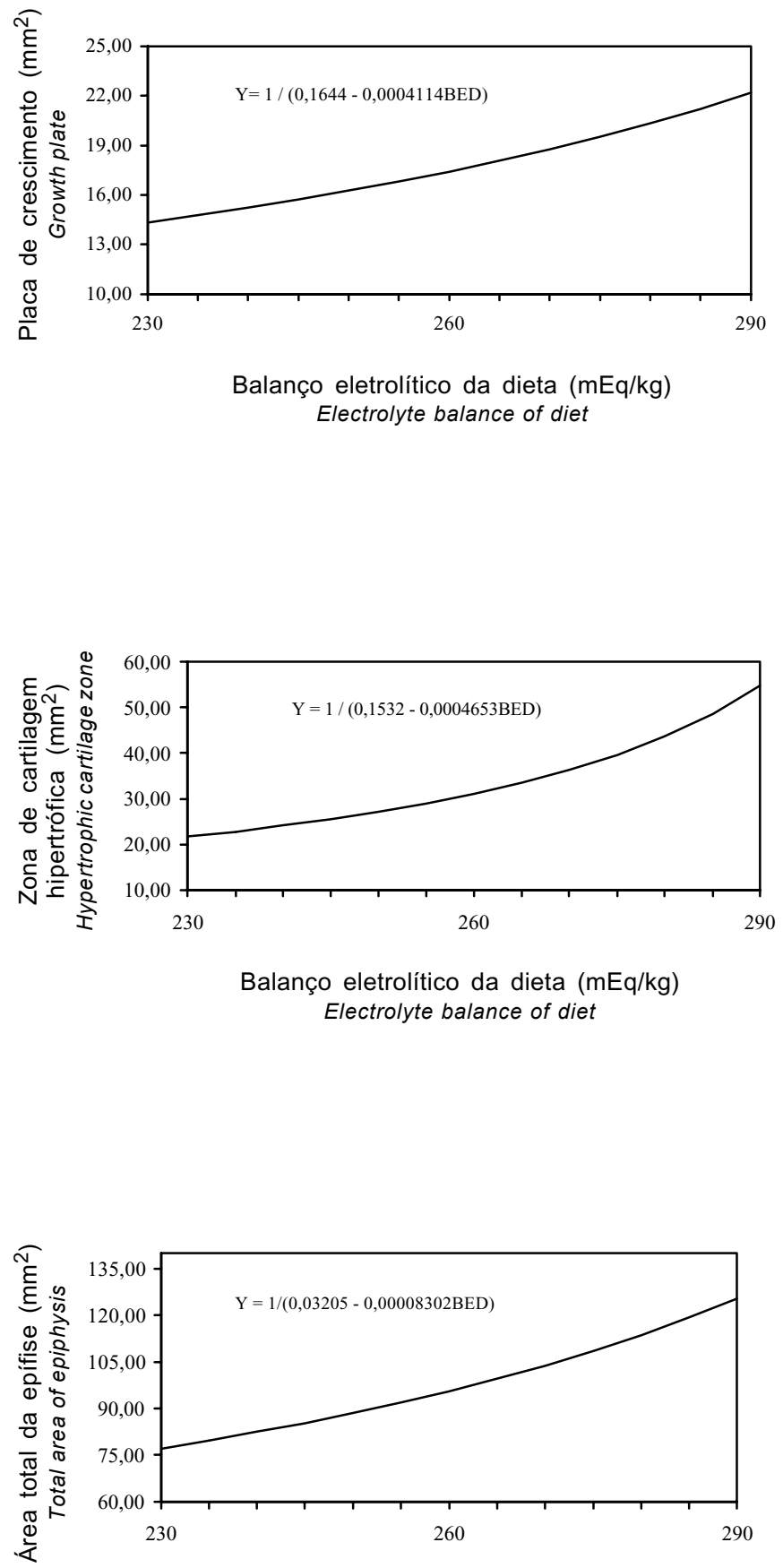

Balanço eletrolítico da dieta $(\mathrm{mEq} / \mathrm{kg})$ Electrolyte balance of diet

Figura 4 - Efeito do balanço eletrolítico, em dietas contendo lasalocida, sobre a placa de crescimento, zona de cartilagem hipertrófica e área total da epífise tibial de frangos de corte aos 21 dias de idade.

Figure 4 - Effect of electrolyte balance of diets with lasalocid on growth plate, hypertrophic cartilage zone and total area of epiphysis of tibial cartilage of broilers chickens in the starting period. respectivamente, sem adição de ionóforos, em dietas com baixos níveis protéicos.

No entanto, observou-se no presente trabalho que, com o aumento do balanço eletrolítico, houve potencialização do lasalocida. Essa potencialização pode ter interferido, indiretamente, no processo normal de calcificação óssea, através da alteração na absorção de minerais, bem como de nutrientes no intestino delgado.

$\mathrm{O}$ efeito dos ionóforos sobre o aumento da permeabilidade das membranas aos íons $\mathrm{H}^{+}$, um fator significativo no equilíbrio ácido-básico (Rutz et al., 1999), poderia ter envolvimento com o aumento das áreas ósseas relacionados com a lesão, no entanto, os parâmetros que poderiam comprovar essa afirmação em relação ao equilíbrio ácido-básico não foram avaliados.

Além disso, segundo a Roche (1994), o lasalocida, ao contrário dos outros ionóforos, é o único que atua nos íons bivalentes $\left(\mathrm{Ca}^{++} \mathrm{e} \mathrm{Mg}^{++}\right)$, sendo o cálcio um dos principais cátions relacionados com a calcificação óssea. Ao se ligar a esses íons, o lasalocida poderia alterar a disponibilidade e bioatividade dos mesmos nos tecidos ósseos pré-calcificados.

Ambos cálcio e fósforo são transportados para a placa de crescimento epifisária ativa, necessitando estar em concentrações adequadas para precipitarem em cristais de hidroxiapatita e completarem o processo de calcificação óssea (Leeson \& Summers, 2001). Com a redução da disponibilidade do cálcio, esse processo poderia ser prejudicado, favorecendo o aumento da área da zona hipertrófica, através da não calcificação dos condrócitos, os quais permaneceriam pré-hipertróficos, levando ao acúmulo de uma massa cartilaginosa não calcificada nessa área, indicando a ocorrência da lesão.

\section{Conclusões}

Nas condições em que o experimento foi realizado, pode-se concluir que as dietas com lasalocida reduziram o desempenho de frangos de corte, sendo que as aves que receberam salinomicina apresentaram maior peso aos 21 dias e não sofreram influência da mesma na incidência de discondroplasia tibial, independentemente do balanço eletrolítico da dieta. No entanto, para o lasalocida, houve aumento das áreas ósseas que caracterizam a discondroplasia tibial, com o aumento do balanço eletrolítico da dieta. 


\section{Literatura Citada}

AUSTIC, R.E.; SMITH, J.B. Interaction of ionophores with nutrients. In: GEORGIA NUTRITION CONFERENCE, 1980, Athens. Proceedings... Athens: The University of Georgia, 1980. GA. 2-10.

BEÇAK, W.; PAULETE, J. Técnicas de citologia e histologia. Rio de Janeiro: Livros Técnicos e Científicos, 1976. 305p.

BERGEN, W.G.; BATES. D.B. Ionophores: their effect on production efficiency and mode of action. Journal of Animal Science, v.58, n.6, p.1465-1483, 1984.

BUSHINSKY, D.A. Stimulated osteoclastic and suppressed osteoblastic activity in metabolic but not respiratory acidosis. American Journal Physiology, v.268, (Cell Physiology), n.37, C80-88, 1995.

BUSHINSKY, D.A.; SESSLER, N.E. Critical role of bicarbonate in calcium release from bone. American Journal Physiology, v.263, (Renal Fluid Electrolyte Physiology), n.32, F.425515,1992

EDWARDS JR., H.M. Studies on the etiology of tibial dyschondroplasia in chickens. Journal of Nutrition, v.114, p.1001-1013, 1984.

ELSASSER, T.H. Potential interactions of ions ionophores drugs with divalent cations and their functions in the animal body. Journal of Animal Science, v.59, n.3, p.845-853, 1984.

FERREIRA, A.J.P.; DELL'PORTO, A. Agentes antiprotozoários In: SPINOSA, H.S.; BERNARDI, M.M.; GONNIAKS, S.L. (Eds.) Farmacologia aplicada à medicina veterinária. 2.ed. Rio de Janeiro: Guanabara Koogan, 1992. p.466-479.

HALLEY, J.T.; NELSON, T.S.; KIRBY, L.K. et al. Effect of altering dietary mineral balance on growth, leg, abnormalities and blood base excess in broiler chicks. Poultry Science, v.66, n.10, p.1684-1692, 1987.

HOOGE, D.M. Influence of dietary electrolytes and their interaction with ionophore coccidiostats on broiler and turkey performance. In: ARKANSAS NUTRITON CONFERENCE, 1995, Arkansas. Proceedings... Arkansas: University of Arkansas, 1995. p.25-50.

HOOGE, D.M. A importância dos eletrólitos. Avicultura Industrial, n.1068, p.20-26, 1999.

HULAN, H.W.; SIMONS, P.C.M.; Van SCHAGEN, P.J.W. et al. Effect of dietary cation-anion balance and calcium content on general performance and incidence of leg abnormalities o broiler chickens. Canadian Journal of Animal Science, v.67, p.165-177, 1987.

JENSEN, L.S. Evaluation of ionophores in nutrition. In: GEORGIA NUTRITION CONFERENCE, 1980, Athens. Proceedings... Athens: The University of Georgia, 1980. GA. 1.

KARUNAJEEWA, H.; BARR, D.A. Influence of dietary electrolyte balance, source of added potassium and anticoccidial agents on the performance of male broilers. British Poultry Science, v.29, p.137-147, 1988.

KRIEGER, N.S.; SESSLER, N.E., BUSHINSKY, D.A. Acidosis inhibits osteoblastic and stimulates activity in vitro. American Journal Physiology, v.262, (Renal Fluid Electrolyte Physiology) n.31, p.442-448, 1992.

LEACH, R.M.; NEISHEIM, M.C. Nutritional, genetic and morphological studies of na abnormal cartilage formation of young chicks. Journal of Nutrition, v.83, p.236-244, 1965.

LEACH, R.M.; NEISHEIM, M.C. Further studies on tibial dyschondroplasia (cartilage abnormality) in young chicks. Journal of Nutrition, v.102, p.1673-1680, 1972.

MONGIN, P.; SAUVEUR, B. Interrelationships between mineral nutrition, acid-base balance, growth and cartilage abnormalities. In: GROWTH AND POULTRY MEAT PRODUCTION, 1977, Edinburgh. Proceedings... Edinburgh: British Poultry Science, 1977. p.235-237.

MONGIN, P. Electrolytes in nutrition: a review of basic principles and practical application in poultry and swine. In: ANNUAL MINESOTTA CONFERENCE, 3., 1980, Illinois. Proceedings... Illinois: IMC, 1980. p.1-15.

MURAKAMI, A.E., GALLI, J.R.; MARTINS, E.N. et al. Efeito do balanço eletrolítico em dietas de baixo conteúdo de proteína no desempenho e na incidência de discondroplasia tibial em frangos de corte. In: CONFERÊNCIA APINCO DE CIÊNCIAS E TECNOLOGIAS AVÍCOLAS, 2000, Campinas. Anais... Campinas: FACTA, 2000. p.40.

NOVILLA, M.N. The veterinary importance of the toxic syndrome induced by ionophores. Veterinary and Human Toxicology, v.34, n.2, p.66-70, 1992.

OVIEDO-RONDON, E.O.; MURAKAMI, A.E.; MARTINS, E.N. et al. Sodium and chloride nutritional requirements for young broiler chickens (1 to 21 days of age). In: POULTRY SCIENCE ASSOCIATION, ANNUAL MEETING AUGUST, 88., 1999, Arkansas. Proceedings... Arkansas: Poultry Science Association, 1999. p.63.

PESTI, G.M.; BAKALLI, R.I.; CERVANTES, H.M. et al. Studies on semduramicin and nutritional responses. 1. Level and source of protein. Poultry Science, v.78, p.102-106, 1999.

PFIZER. Anticoccidiano PFIZE; Coxistac premix - Salinomicina 6\%. In: SIMPÓSIO INTERNACIONAL SOBRE COCCIDIOSE, 19., 1994, Santos. Anais... Santos: FACTA, 1994. p.167-172.

RIDDEL, C. Studies on the pathogenesis of tibial dyschondroplasia in chickens. II. Growth rate of long bones. Avian Diseases, v.19, n.3, p.490-496, 1975.

ROSTAGNO, H.S.; ALBINO, L.F.T.; DONZELE, J.L. et al. Tabelas brasileiras para aves e suínos: composição de alimentos e exigências nutricionais.Viçosa, MG: Universidade Federal de Viçosa, 2000. 141p.

ROCHE. Anticoccidiano ROCHE: Avatec. In: SIMPÓSIO INTERNACIONAL SOBRE COCCIDIOSE, 20., 1994, Santos. Anais... Santos: FACTA, 1994. p.173-176.

ROSADO, A.M.S. Efeito de coccidiostáticos ionóforos sobre o desempenho e o balanço de eletrólitos em frangos de corte. Viçosa, MG: Universidade Federal de Viçosa, 1988. 75p. Dissertação (Mestrado em Zootecnia) - Universidade Federal de Viçosa, 1988.

RUÍZ-LOPES, B.; RANGEL-LUGO, M.; AUSTIC, R.E. Effects of selected minerals on acid-base balance and tybial dyschondroplasia in broilers chickens. Poultry Science, v.72, p.1693-1704, 1993.

RUTZ, F.; XAVIER, E.G.; ROLL, V.F.B. et al. Interação entre nutrição e agentes anticoccidianos. In: SIMPÓSIO INTERNACIONAL SOBRE NUTRIÇÃO DE AVES, 1999, Campinas. Anais... Campinas: 1999. p.199-217.

SAUVEUR, B.; MONGIN, P. Tibial dyschondroplasia, a cartilage abnormality in poultry. Annales de Biologie Animale Biochimie, Biophysique, v.18, p.87-98, 1978.

SPRAGUE, S.M.; KRIEGER, N.S.; BUSHINSKY, D.A. Greater inhibition of in vitro bone mineralization with metabolic than respiratory acidosis. Kidney Int, v.46, n.4, p.1199-1206, 1994.

R. Bras. Zootec., v.33, n.1, p.135-145, 2004 
THORP, B.H.; DUCRO, B.; WHITEHEAD, C.C. Avian tibial dyschondroplasia: the interaction of genetic selection and dietary 1,25-dihydroxycholecalciferol. Avian Pathology, v.22, p.311-324, 1993.

VIEIRA, S.L.; RUTZ, F. Carboidratos e proteínas: digestão e absorção In: MACARI, M.; FURLAN, D.L.; GONZALEZ, E. (Eds.) Fisiologia aviária aplicada a frangos de corte. 2.ed. ampliada. Jaboticabal: Universidade Estadual Paulista, 2002. p.125-139.

WHEELHOUSE, R.K.; GROVES, B.I. Effects coccidiostats and dietary protein on performance and water consumption in broiler chickens. Poultry Science, v.64, n.5, p.979-85, 1985.
WHITEHEAD, C.C. Bone biology and skeletal disorders in poultry. Oxfordshire: Carfax Publishing Company Abingdon, 1992. 380p.
Recebido em: 27/08/02

Aceito em: 27/05/03 